\title{
3 Alternativen in der Sozialen Erkenntnistheorie
}

\subsection{Philosophie, Geschichte und Soziologie der Wissenschaft}

Die neopositivistische Konzeption von Wissenschaft und die entsprechenden Maßstäbe für epistemische Güte haben sich als zu strikt erwiesen. Ein stärkerer Einbezug der Wissenschaftsgeschichte hat gezeigt, dass auch historische Fälle, die gemeinhin als Meilensteine der Wissenschaft betrachtet werden (wie die kopernikanische Wende oder die chemische Revolution), mit diesen Maßstäben nicht angemessen beschrieben werden können. Zudem haben systematische Argumente darauf hingewiesen, dass Logik und Erfahrung allein für Urteile über Geltungsansprüche nicht ausreichen. Auf dieser Grundlage wird nun weithin anerkannt, dass entsprechende Urteile oft nicht eindeutig sind und Entscheidungen über die Akzeptanz oder Zurückweisung von Theorien sowohl komplexer als auch ergebnisoffener, als ein verifikationistischer Empirismus zulässt.

According to earlier logicist views, science consists primarily of law-like assertions, and these are assessed in a relatively definitive way by means of a set of rules. The newer stress is on science as theory, and on the variety of criteria, none of them coercive, which govern theory appraisal. (McMullin 1984, S. 145)

Die Reaktionen auf dieses prinzipielle Maß an Unsicherheit wissenschaftlichen Wissens und die Einschätzungen seiner Reichweite sind allerdings so divers wie konfliktträchtig. Etwas vereinfacht gesagt, lässt sich eine Opposition zwischen der Wissenschaftsphilosophie und verschieden konstruktivistischen Ansätzen mit vorwiegend sozialwissenschaftlichem Hintergrund konstatieren. In der Wissenschaftsphilosophie gibt es gravierende Vorbehalte und Einwände dagegen, die Möglichkeit einer normativen Betrachtung von Wissenschaft aufzugeben. Eine solche sollte einerseits von der tatsächlichen Wissenschaft einlösbar sein, andererseits zwischen guter und schlechter Wissenschaft differenzieren (und so die Grundlage für Evaluierungen von Geltungs- und Objektivitätsansprüchen bilden) können.

Larry Laudan und Imre Lakatos sind prominente Beispiele für metamethodologische Ansätze, die zum einen die Wissenschaftsgeschichte 
stärker reflektieren, zum anderen Kriterien für die Rationalität wissenschaftlichen Wandels formulieren. Beide inkorporieren die Einsicht, dass Hypothesen nicht einzeln mit der Empirie abgeglichen werden können, wenden sich jedoch gegen die irrationalen Implikationen (oder Interpretationsmöglichkeiten) des Kuhnschen Paradigmenansatzes. Lakatos plädiert dafür, Forschungsprogramme auf der Grundlage ihrer Progressivität zu evaluieren, die sich vor allem an dem Treffen erfolgreicher, neuer Vorhersagen bemisst (vgl. Lakatos 1978a). Laudan hingegen bewertet die Progressivität von Forschungstraditionen anhand der Anzahl und Frequenz hervorgebrachter Problemlösungen (vgl. Laudan 1977).

Beide Ansätze räumen dabei trotz ihrer Normativität der tatsächlichen Geschichte der Wissenschaft eine wichtige Rolle ein und verwenden historische Fallstudien zur Untermauerung ihrer Argumentation. Allerdings ist dieses Verhältnis der Wissenschaftsphilosophie zur Wissenschaftsgeschichte aufgrund ihrer normativen respektive deskriptiven Ausrichtung nicht unproblematisch. So betrachtet etwa Lakatos die Wissenschaftsgeschichte als Prüfmöglichkeit normativer Ansätze insofern, als deren Qualität auch dadurch bestimmt werden soll, wie weite Bereiche dieser Geschichte sie als rational auszuzeichnen vermögen, und ob sie eine rationale Rekonstruktion von Fällen erlauben, die gemeinhin als wissenschaftliche Erfolge betrachtet werden (vgl. Lakatos 1978b). Eine Rationalität dieser Fälle wird demnach vorausgesetzt, was zu dem Vorwurf der Zirkularität geführt hat. Hilfreich ist an dieser Stelle eine von McMullin eingeführte Unterscheidung zwischen der impliziten Rationalität historischer Fallbeispiele, die sich auf die tatsächlich zeitgenössisch verwendeten Maßstäbe von Rationalität bezieht, und zugeschriebener Rationalität, die begründet, warum diese Episoden auch nach heutigen Maßstäben als wissenschaftliche Erfolge gelten können. Solange zwischen diesen Formen der Rationalitätszuschreibung differenziert werde, sei es durchaus legitim, die Möglichkeit der Zuschreibung bestimmter Rationalitätsmaßstäbe als indirekte Unterstützung für diese Maßstäbe zu betrachten. Problematisch sei nur, die gemachten Zuschreibungen als historische Erklärung auszugegeben und als These über die implizite Rationalität der Wissenschaftsgeschichte zu präsentieren (vgl. McMullin 1984, S. 136-141).

Hierbei bleibt allerdings unklar, wo die Grenzen der Rekonstruktion liegen, wie weit diese von der historischen Evidenz abweichen darf und 
wie der Gefahr begegnet werden kann, dass die Geschichte schlicht entsprechend der jeweiligen metamethodologischen Auffassung interpretiert wird, wodurch diese sich wiederum selbst bestätigen würde. Laudan grenzt sich deshalb von einer Abgleichung philosophischer Ansätze mit rationalen Rekonstruktionen $\mathrm{ab}$, obschon auch für ihn die Wissenschaftsgeschichte eine wichtige Rolle in Bezug auf die Prüfung der Wissenschaftstheorie spielt. Er geht davon aus, dass wir bestimmte Intuitionen über gute Wissenschaft teilen und uns auf eine Grundmenge großer wissenschaftlicher Erfolge einigen können. Genau diese wiederum müssten durch eine adäquate Wissenschaftstheorie auch als solche erfassbar und erklärbar seien. Hierbei werden offensichtlich ebenfalls Intuitionen und philosophische Ansichten in die Interpretation der Geschichte hineingetragen; dies sei unvermeidlich, Ziel sei jedoch eine möglichst tatsachengetreue Darstellung (vgl. Laudan 1977, Kap. 5).

Zwei Punkte sind in Bezug auf die Relation von Wissenschaftsphilosophie und -geschichte festzuhalten. Erstens lässt sich eine gewisse Zirkularität nicht umgehen, wenn normative philosophische Ansätze durch bestimmte Episoden der Geschichte unterstützt oder unterminiert werden sollen, da auch in der Geschichte keine theoriefreie Interpretation möglich ist. Um überhaupt Relevanzentscheidungen treffen zu können, braucht der Wissenschaftshistoriker ein gewisses Vorverständnis davon, was Wissenschaft ist, und inkorporiert so möglicherweise bestimmte philosophische Annahmen. Dieser Zirkel muss jedoch nicht zwingend geschlossen sein, sondern kann auch spiralförmig gedacht werden. Buchdahl (1987) etwa betrachtet dieses Verhältnis als eines der quasi-dialektischen Koevolution, in welchem die Philosophie und die Geschichte jeweils offen für die Kritik der anderen Disziplin sind und sich so gemeinsam weiterentwickeln können. Zweitens ergeben sich keine direkten normativen Schlussfolgerungen aus deskriptiver Geschichte. Bestimmte Kriterien werden vielmehr immer schon vorausgesetzt, wie etwa, dass die Meilensteine der Wissenschaftsgeschichte wirkliche Erfolge und rational erklärbar sind (eine Annahme, die zumindest sehr plausibel scheint).

Auf der anderen Seite wird jedoch gerade die hier verwendete Prämisse einer Rationalität der großen Erfolgsgeschichten der Wissenschaft von den verschiedenen sozialkonstruktivistischen Ansätzen nicht geteilt, weshalb der betreffende Konflikt so leicht nicht beizulegen ist (vgl. Brown 
2001, S. 71 ff.). Der Sozialkonstruktivismus beinhaltet eine Reihe unterschiedlicher Ansätze, die differenziert wiederzugeben hier nicht möglich ist (für einen ausführlicheren Überblick vgl. z. B. Hess 1997). ${ }^{36}$ Brown (2001) unterscheidet grob zwischen einer nihilistischen und einer naturalistischen Ausrichtung. Unter erstere subsumiert er verschiedene postmoderne und dekonstruktivistische Ansätze (wie etwa Lyotard, Lacan oder Derrida), die den Objektivitätsanspruch der Wissenschaft gänzlich zurückweisen. Der naturalistische Flügel geht hingegen selbst mittels wissenschaftlicher Methoden vor, um Wissenschaft zu beschreiben. Entscheidend ist, dass hier, im Gegensatz etwa zu einer Wissenschaftssoziologie in der Tradition Mertons, ${ }^{37}$ auch der Inhalt wissenschaftlichen Wissens zu einem Gegenstand der Forschung wird und sozialwissenschaftlich erklärt werden soll.

Eine prominente Rolle hat in diesem Kontext vor allem das Strong Programme in the Sociology of Knowledge der Edinburgh School (im Folgenden: SSK) gespielt, für das David Bloor (1976) die folgenden programmatischen Prinzipien aufstellte: Kausalität, d. h. Ziel sind kausale Erklärungen wissenschaftlicher Überzeugungen; Impartialität, d. h. diese Erklärungen sollten unabhängig von Aussagen über die Berechtigung von Geltungsansprüchen dieser Überzeugungen sein; Symmetrie, d. h. akzeptierte Überzeugungen erfordern dieselbe Art kausaler Erklärung wie zurückgewiesene; und schließlich Reflexivität, d. h., diese Prinzipien sollen auch auf die Soziologie wissenschaftlichen Wissens selbst angewendet werden.

Kontrovers ist dabei vor allem die Frage, ob rationale Gründe Ursachen für Überzeugungen darstellen können, was von den Vertretern des SSK verneint wird. Diese gehen davon aus, dass die Ursachen von Theoriewahlentscheidungen in sozialen Faktoren wie z. B. politischen Interes-

${ }^{36}$ Die folgende Diskussion bezieht sich zudem nur auf einen epistemologischen Sozialkonstruktivismus, nicht auf ontologische Thesen.

${ }^{37}$ Merton beschreibt den sozialen Kontext als Erfolgsbedingung der Wissenschaft und konzentriert sich dabei vor allem auf die bekannte Reihe institutioneller Werte (Kommunitarismus, Universalismus, Uneigennützigkeit, organisierte Skepsis). Die Einflüsse dieser Werte sind jedoch mit dem Wertfreiheitsideal kompatibel, da sie für die Evaluierung von Geltungsansprüchen nicht von Bedeutung sein sollen (vgl. z. B. Merton 1942). 
sen bestehen, ${ }^{38}$ während die meisten Wissenschaftsphilosophen an der kausalen Relevanz guter Gründe festhalten. Viel Kritik hat zudem auch das Symmetrieprinzip erfahren. Exemplarisch sei hier Laudan genannt, der dafür argumentiert, dass eine Erklärung mittels sozialer Faktoren nur dann berechtigt sei, wenn es keine rationale Erklärung der betreffenden Überzeugungen gebe (eine rationale Erklärung, sofern möglich, einer sozialen also immer vorzuziehen sei; vgl. Laudan 1977, S. 201 ff.). Wie McMullin konstatiert, beruht dieser Einwand nicht nur auf der Voraussetzung eines transhistorischen Rationalitätsstandards, sondern zusätzlich auf einer ebenso problematischen (und mit dem SSK geteilten) Dichotomie in Bezug auf rationale und soziale Faktoren. Ihm zufolge müssen die soziale und die rationale Seite nicht in exklusiver Opposition zu einander stehen, sondern

${ }^{38}$ Im Gegensatz dazu postuliert beispielsweise Latours Akteur-Netzwerk-Theorie, dass die kausalen Einflüsse keineswegs unidirektional sind, wobei Latour einen Begriff des (kausal relevanten) Aktanten benutzt, der nicht mehr zwischen belebter und unbelebter Materie differenziert und dafür kritisiert wird, letzterer Handlungen zuzuschreiben. Zusätzlich zu diesen makrosoziologischen und eher historisch ausgerichteten Ansätzen gibt es auch eine Reihe mikrosoziologischer Arbeiten, wobei insbesondere die Laborstudien von Knorr-Cetina (und anderen) zu erwähnen sind. Hier wird durch eine kleinteilige Beobachtung von Forschungsabläufen nachzuweisen versucht, wie soziale Faktoren und individuelle Interessen Einfluss auf die Resultate nehmen und gleichzeitig in diesen Resultaten unsichtbar gemacht werden.

Eine entscheidende Größe in diesen Debatten waren nicht zuletzt verschiedene feministische Ansätze; da sich diese mit den dargestellten Standpunkten zum Teil überschneiden und ebenfalls keine einheitliche Position bilden, werde ich sie hier nicht gesondert darstellen. Im Rückgang auf Harding (1986) unterscheidet man drei Arten feministischer Erkenntnistheorie: feminist empiricism, der androzentrische Verzerrungen als empirisch inadäquat kritisiert und an den herkömmlichen Maßstäben guter Wissenschaft festhält; standpoint epistemologies, die von der Perspektivgebundenheit wissenschaftlichen Wissens sowie der epistemologischen Überlegenheit politisch marginalisierter Perspektiven ausgehen, und feminist postmodernism, welcher die epistemische Autorität der Wissenschaft insgesamt bezweifelt. In dieser Arbeit werde ich vor allem auf die Position Helen Longinos eingehen, die insofern zwischen diesen Kategorien steht, als sie einerseits von einem empiristischen Ansatz ausgeht, andererseits jedoch die Perspektivgebundenheit wissenschaftlichen Wissens betont (ohne dabei die epistemologische Bedeutung einzelner Perspektiven zu hierarchisieren).

Darüber hinaus gibt es noch eine Vielzahl von Arbeiten in den Science and Technology Studies, auf die einzugehen hier nicht der Raum ist; vgl. auch dazu Hess (1997) für eine erste Annäherung. 
können sich durchaus ergänzen. Zumindest aber sei nicht a priori, sondern nur mittels detaillierter Fallstudien entscheidbar, ob die Wissenschaftsgeschichte soziale oder rationale Erklärungen erfordere (vgl. McMullin 1984, S. 151 f.).

Mehr als dieser oberflächliche Blick kann hier auf die verschiedenen sozialkonstruktivistischen Ansätze nicht geworfen worden, vor allem da sie im Hinblick auf die Frage, wie Objektivität ohne Wertfreiheit zu denken ist, durch ihre grundsätzliche Ablehnung wissenschaftlicher Objektivitätsansprüche nicht hilfreich sind. Zudem wurden sie bereits häufig kritisiert und offenbaren verschiedene gravierende Probleme. Wie kann auf dieser Grundlage der so evident erscheinende Erfolg der empirischen Wissenschaften erklärt werden? Wie werden aus sozialen Einflüssen komplexe Theorien? Wie ist der Ausschluss rationaler Gründe aus der Menge möglicher Kausalfaktoren für die Akzeptanz von Theorien zu etablieren? Die dargestellte logische Lücke, welche die Unterdeterminierungsthese eröffnet, wird in sozialkonstruktivistischen Argumentationen häufig als Legitimation eines anything goes verstanden. Dazu ist zu sagen, dass hier für Interpretationen ein mögliches Kontinuum von ,nicht vollständig logisch determiniert" bis „vollkommen unbestimmt" besteht. Unterdeterminierung allein zeigt nicht, dass Empirie und Rationalität überhaupt keine Rolle spielen. Um Überreaktionen zu vermeiden, sollte zwischen der logischen Unterdeterminierung von Hypothesen durch Beobachtungen und dem Spielraum bei der tatsächlichen Beurteilung von Hypothesen unterschieden werden (siehe auch Kapitel 6).

Zusammengefasst negieren die sozialkonstruktivistischen Positionen, dass das Wertfreiheitsideal realisierbar im Sinne einer Annäherung an eben dieses ist und deshalb, dass dieses Ideal seine epistemische Funktion erfüllt, Objektivität zu ermöglichen. Dies wiederum impliziert die Unmöglichkeit einer fruchtbaren politischen Funktion im Sinne von Aufklärung; im Gegenteil führe die (nur) vorgebliche Erfüllung der epistemischen Funktion zu einer Festigung bestehender Machtverhältnisse. Sowohl die Kohärenz dieser Kritik als auch das Fehlen einer konstruktiven Alternative, die es erlauben würde, Wissenschaft von anderen Modi der Glaubensproduktion und gute von schlechter Wissenschaft zu unterscheiden, sind dabei jedoch als hoch problematisch einzustufen. 
Insgesamt scheint diese soziale Überdeterminierung ebenso unplausibel wie die neopositivistische Überidealisierung. Im SSK wird den sozialen Aspekte wissenschaftlicher Entscheidungen die exklusive explanatorische Funktion zugeschrieben, welche in der neopositivistischen Wissenschaftstheorie der Empirie und Logik und in metamethodologischen Ansätzen etwas weiter gefassten Rationalitätskriterien zukam. Daraus ergibt sich eine Opposition soziologischer und epistemologischer Ansätze, die sich als wenig fruchtbar erwiesen hat, was einen der Ausgangspunkte der Sozialepistemologie konstituiert. Unter diesem Stichwort lassen sich eine Reihe von wissenschafts- und erkenntnistheoretischen Ansätzen zusammenfassen, die seit etwa zwei Jahrzehnten zunehmend an Bedeutung gewinnen und sich durch die Suche nach einem dritten Weg zwischen idealisierten philosophischen Rationalitätsentwürfen und empirisch arbeitenden Skeptikern auszeichnen. Dabei stehen eine Reihe verschiedener Fragestellungen im Mittelpunkt (vgl. dazu auch Wilholt 2007). Ein Beispiel ist das Problem der cognitive agency, d. h. die Frage danach, ob der (wissenschaftliche) Erkenntnisprozess sich prinzipiell auf Individuen reduzieren lässt oder aber essentiell sozialer Natur ist. Selbst wenn an einer prinzipiellen Reduzierbarkeit festgehalten wird, steht jedoch außer Frage, dass die heutige Wissenschaft zum allergrößten Teil nicht das Ergebnis der Arbeit Einzelner ist, sondern komplexe soziale Zusammenhänge voraussetzt. Eine weitere zentrale Thematik der Sozialepistemologie liegt daher darin, diese sozialen, politischen und institutionellen Rahmenbedingungen in ihrer wissenschaftstheoretischen Bedeutung zu reflektieren. Dabei zielt sie vor allem auch darauf zu erklären, wie wissenschaftliches Wissen in einem sozialen Rahmen produziert wird, ohne deshalb seine epistemische Autorität zu verlieren, d. h. es geht um die Vereinbarkeit normativer philosophischer Konzepte wie Rationalität und Objektivität mit dem Vorhandensein sozialer Einflüsse. $\mathrm{Zu}$ den diesbezüglich diskutierten Einflüssen gehört dabei insbesondere auch die mögliche Wertbeladenheit der Wissenschaft.

In den nächsten Unterkapiteln soll ein Überblick über zwei besonders einflussreiche Ansätze innerhalb der sozialen Erkenntnistheorie gegeben werden - Kitchers Well-Ordered Science (im Folgenden: WOS) und Longinos Social Value Management (im Folgenden: SVM). Beide kritisieren nicht nur die Vorstellung einer wertfreien Wissenschaft, sondern for- 
mulieren auch jeweils ein alternatives normatives Ideal. Während diese Positionen zwar die Prämisse einer wissenschaftstheoretischen Relevanz des sozialen Kontextes von Wissenschaft teilen, unterscheiden sie sich erheblich in Bezug auf die genauere argumentative Bestimmung dieser Relevanz und insbesondere auch auf die Einschätzung der Reichweite möglicher Werteinflüsse in der Wissenschaft.

\subsection{Philip Kitcher: Well-Ordered Science}

Philip Kitcher entwickelt sein Ideal einer demokratisch ausgerichteten und sozial verantwortlichen Wissenschaft in Science, Truth, and Democracy (2001) ausgehend von der Annahme, dass die aktuelle Opposition im Hinblick auf die Reichweite sozialer Einflüsse in der Wissenschaft einen mittleren Weg zwischen sozialkonstruktivistischen und naiv wissenschaftsgläubigen Ansätzen erfordere. Letztere operierten auf Grundlage der Annahme, dass Wissenschaft dem einen übergeordneten und intrinsisch wertvollen Ziel folge, Wissen über die Welt zu erlangen, und auf diesem Weg sehr erfolgreich sei. Als Bedingung dieses Erfolgs werde jedoch die Freiheit der Wissenschaft betrachtet. Diese Freiheit sei als Freiheit von Einflüssen sozialer Werte oder politischer Kräfte zu verstehen und beziehe sich zum einen auf die Ausrichtung von Forschung, zum anderen auf die Bewertung von Geltungsansprüchen. Während Einflüsse auf die AgendaSetzung die Effizienz der Wissenschaft verminderten (siehe oben zum Reinheitsaspekt des Wertfreiheitsideals), untergrüben Einflüsse auf den Rechtfertigungskontext die Objektivität der Wissenschaft und damit ihren Anspruch auf epistemische Autorität. Dies entspreche wiederum dem Ziel der gegnerischen Seite: wissenschaftliche Objektivität auf der Basis der Unterdeterminierungsthese als Mythos auszuweisen, der den politischen Interessen einer mächtigen Elite diene (vgl. Kitcher 2001, Kap. 1).

Kitcher nimmt zwischen diesen beiden Extremen insofern eine Zwischenposition ein, als er zwar an der Forderung nach einer Wertfreiheit der Rechtfertigung festhält, die Idee einer Reinheit der Agenda-Setzung jedoch als verfehlt kritisiert. Der erste Punkt basiert vor allem auf seiner 
Zurückweisung der Unterdeterminierungsthese. Diese unterteilt er in drei Versionen mit unterschiedlicher Reichweite: erstens transiente Unterdeterminierung, die sich auf die Situation bezieht, dass eine Theoriewahlentscheidung aktuell durch die verfügbare Evidenz unterbestimmt ist; zweitens permanente Unterdeterminierung, die vorliegt, wenn diese Entscheidung auch durch alle zukünftige Evidenz nicht determiniert werden kann; und drittens globale Unterdeterminierung, womit er die These bezeichnet, dass sämtliche Theoriewahlentscheidungen aufgrund der Möglichkeit empirisch äquivalenter Alternativen immer unterbestimmt bleiben (also alle Wissenschaft permanent unterdeterminiert ist).

In Bezug auf den ersten Fall konstatiert er, diese Situation sei zwar allgegenwärtig, jedoch für den Objektivitätsanspruch der Wissenschaft irrelevant: "Transient underdetermination is familiar and unthreatening" (ebd., S. 30). Den Grund für diese Einschätzung führt er nicht weiter aus; vermutlich basiert sie auf der Annahme, dass die betreffenden Theoriewahlentscheidungen durch zukünftige Belege determiniert werden können und diese Entscheidungen entsprechend bis zu diesem Zeitpunkt auszusetzen sind. ${ }^{39}$ Des Weiteren räumt Kitcher zwar ein, dass die Möglichkeit permanenter Unterdeterminierung bestehe, betrachtet diese aber nicht als prinzipielles Problem, da die meisten Konflikte zwischen Alternativen letztlich doch einer (evidenzbasierten) Lösung zugeführt werden könnten, wie unter anderem die Wissenschaftsgeschichte zeige. Globale Unterdeterminierung zieht er generell in Zweifel. Zum einen sei fraglich, ob es tatsächlich immer echte Alternativen gebe; zum anderen sei die These einer globalen Unterdeterminierung inkonsistent mit der Annahme, die durch die Erfahrung unterbestimmten Entscheidungen würden durch soziale Einflüsse und politische Präferenzen determiniert. Annahmen über den Zusammenhang der jeweiligen Theorien mit bestimmten Werten oder sozialen Konsequenzen seien von der globalen These gleichfalls betroffen und

\footnotetext{
${ }^{39}$ Diesem Agnostizismus-Argument werden wir im Verlauf der Arbeit noch mehrfach begegnen. Es besagt, dass in Fällen, in denen ohne Rückgriff auf außerwissenschaftliche Werte keine Entscheidung über den Geltungsanspruch einer Theorie getroffen werden kann, dieses Urteil auszusetzen und auf einen weiteren Fortschritt der Wissenschaft zu warten ist (etwa auf neue empirische Daten). Es wird zu diskutieren sein, ob eine solche Aussetzung des Urteils immer möglich ist.
} 
vermöchten daher eine solche Determinierung ebenso wenig zu leisten (vgl. ebd., Kap. 4).

Anzumerken ist, dass Kitchers Zurückweisung einer Unterdeterminierung sich vorrangig gegen deren kontrastive Fassung richtet, d. h. sich auf die Frage bezieht, ob die Wahl zwischen verschiedenen Theorien anhand empirischer Evidenz möglich ist. In Bezug auf die Problematik holistischer Unterdeterminierung, welche die Frage der Isolierbarkeit zu überprüfender Hypothesen und ihren Zusammenhang mit bestimmten empirischen Konsequenzen betrifft, postuliert er, dass die Ableitung dieser Konsequenzen aus Theorien wertfrei möglich sei: "it is at least possible to take the first step and to identify the predictions and interventions that flow from a theory without the intrusion of value judgements" (ebd., S. 30). ${ }^{40}$

Auf der Grundlage seiner Zurückweisung des Unterdeterminierungsproblems vertritt Kitcher einen moderaten wissenschaftlichen Realismus ${ }^{41}$ und geht davon aus, dass Theoriewahlentscheidungen wertfrei möglich seien, was wiederum die Grundlage des wissenschaftlichen Anspruches auf Objektivität bilde:

[T] he resolution of scientific debate on the basis of evidence is possible. The ideal of objectivity need not be dismissed as a fond delusion. Hence there is no basis for believing that value judgments inevitably enter into our appraisal of which of a set of rival hypotheses (if any) is approximately correct. (Ebd., s. 41)

Kitcher hält folglich an dem herkömmlichen Objektivitätsverständnis fest, welches die Wertfreiheit von Theoriewahlentscheidungen voraussetzt. Während er damit genau den Aspekt der Wertfreiheit verteidigt, der oben

40 Argumente $\mathrm{zu}$ holistischer und kontrastiver Unterdeterminierung (und damit die Haltbarkeit von Kitchers diesbezüglicher Position) werden in Kapitel 6 respektive 7 noch genauer diskutiert; an dieser Stelle soll zunächst ein Überblick über die Grundlagen von WOS gegeben werden.

${ }^{41}$ Auf Kitchers Argumentation für einen wissenschaftlichen Realismus soll an dieser Stelle nicht genauer eingegangen werden, da mich vornehmlich seine Haltung zur Frage der Wertfreiheit der Wissenschaft interessiert (die hier unabhängig von realistischen Voraussetzungen diskutiert werden soll). Er behandelt einige antirealistische Argumente, wobei er erstens die empiristische Distinktion zwischen Beobachtbarem und Nicht-Beobachtbarem als kontingent und kontextabhängig bezeichnet, sich zweitens angesichts von Laudans pessimistischer Meta-Induktion auf die Wahrheit bestimmter Teile von Theorien zurückzieht und drittens sozialkonstruktivistische Positionen als inkohärent beschreibt (vgl. ebd., Kap. 2). 
als entscheidend für das zeitgenössische Ideal beschrieben wurde (i.e., Theoriewahlentscheidungen dürfen nicht auf außerwissenschaftliche Werte rekurrieren), versucht er andererseits, die Agenda-Setzung stärker an demokratischen Prinzipien auszurichten und damit explizit an gesellschaftliche Wertdebatten zu koppeln. Auch wenn die von ihm identifizierte Wertbeladenheit der Ausrichtung von Forschung bereits seit längerem mehrheitsfähig ist, so ist es doch Kitchers Verdienst, den Entdeckungszusammenhang stärker in die wissenschaftsphilosophische Diskussion einzubeziehen und systematisch als in einem sozialen Kontext stehend und normativ relevant zu reflektieren. Seine Kritik an der Forderung nach einer Autonomie der Ausrichtung richtet sich dabei vor allem gegen die Prämisse, Wissenschaft verfolge ein einziges und eindeutiges Ziel: die Erkenntnis der Wahrheit. Diese Zielformulierung reiche nicht aus, um über die thematische Ausrichtung von Forschung zu entscheiden. Wissenschaft suche keineswegs nach einer unendlichen Menge (auch noch so trivialer) Wahrheiten, sondern nach signifikanten Wahrheiten. Die Bestimmung dieser Signifikanz sei relativ zu spezifischeren Zielen und damit wertbeladen. Zwar sei es durchaus möglich, zwischen epistemischer Signifikanz und praktischer Signifikanz zu unterscheiden, auch erstere sei aber nicht kontextfrei zu denken. Entscheidend ist an dieser Stelle Kitchers Konzept der Signifikanzgraphen. Dieses bringt zum Ausdruck, dass selbst epistemische Signifikanz von der historischen Entwicklung des jeweiligen Forschungsbereichs abhängig ist. Was aktuell aus einer wissenschaftlichen Perspektive heraus als interessante Frage erscheine, sei auch dadurch bestimmt, welche Erkenntnisse bereits erlangt und welche Bereiche bisher ausgeklammert wurden. Dies wiederum könne auch durch soziale oder praktische Belange in der Vergangenheit beeinflusst worden sein (vgl. ebd., Kap. 6 und 7).

Der angenommene Einfluss der verschiedenen Ziele, die durch Wissenschaft verfolgt werden können, reicht dabei über die reine Themenwahl hinaus. Forschung sei nicht einfach ein direkter Spiegel der Natur, sondern nähere sich dieser immer aus einer bestimmten Perspektive. Deshalb sei beispielsweise auch die Identifizierung relevanter Objekte sowie ihre Klassifizierung und Beschreibung im Hinblick auf die verfolgten Zwecke zu sehen. Die entstehenden Taxonomien prägten wiederum die spätere Sicht 
auf Gegenstandsbereiche, da andere Möglichkeiten dann nicht entwickelt würden:

All kinds of considerations, including moral, social, and political ideals, figure in judgments about scientific significance, and hence in the evolution of significance graphs. Inquiries that appeal to us today, and that we characterize as epistemically significant, sometimes do so because of the practical projects our predecessors pursued in the past. With our eyes focused on the present, it's easy to deny that these inquiries are in any way connected with broader values. A longer view would reveal that the questions we pose, the apparatus we employ, the categories that frame our investigations, even the objects we probe, are as they are because of the moral, social, and political ideals of our predecessors. (Ebd., S. 86)

Unsere Ziele prägen demnach unsere Kategorien, und zwar auch in einem historisch relevanten Sinn derart, dass spätere Forschung - selbst wenn sie abweichende Ziele verfolgen sollte - diese Prägung weiterhin inkorporiert. Darüber hinaus beeinflussen diese Ziele auch die Standards der Theoriebewertung, da empirische Adäquatheit nicht ausreiche, deren Güte zu bestimmen. Zusätzlich müssten diese Theorien Kriterien erfüllen, die sich daraus ableiteten, in welcher Hinsicht wir ihnen Signifikanz zuschrieben. Kitcher vergleicht diesbezüglich Theorien mit Karten, die sich je nach Zweck unterscheiden, dennoch aber, eben im Hinblick darauf, wie gut oder schlecht sie diesen Zweck erfüllen, evaluierbar sind (an dieser Stelle sei an McMullins Unterscheidung von valuing und evaluation erinnert). In Bezug auf die Ziele von Forschung vertritt Kitcher damit einen Pluralismus, der sich auf Inhalt und Bewertung von Theorien auswirkt. Er geht jedoch davon aus, dass diese unterschiedlichen Karten ineinander übersetzbar sind und nicht miteinander inkompatibel sein dürfen (vgl. ders. 2002a, 2002b). ${ }^{42}$

Die Abhängigkeit wissenschaftlicher Signifikanz - auch epistemischer Signifikanz - von bisherigen und aktuellen Zielen und der vorhergehenden Entwicklung eines Forschungsbereichs bildet einen Grundpfeiler von Kitchers Argumentation gegen die Freiheit der Agenda-Setzung.

\footnotetext{
${ }^{42}$ Damit vertritt er eine schwache Pluralismusthese im Gegensatz zu Longino, welche die Möglichkeit inkompatibler, jeweils akzeptierbarer Theorien für gegeben hält und kritisiert, dass auch Kitchers Auffassung kontextabhängiger Signifikanzzuschreibungen und entsprechender Evaluierungsstandards diese Möglichkeit impliziere (vgl. Longino 2002b, 2002c; zur Diskussion dieser Problematik siehe Kapitel 7).
} 
Entscheidungen, die wir heute in Bezug auf die Auswahl von Themen und Hypothesen treffen, sind zwar durch diese Historizität der Wissenschaftsentwicklung nicht determiniert, wohl aber in entscheidender Weise geprägt und eingeschränkt. Jede Wahl zwischen verschiedenen Möglichkeiten nimmt Einfluss auf zukünftige Wahlmöglichkeiten und ist nicht einfach rückgängig zu machen. Eine solche Wahl ist aber aufgrund der Begrenztheit der zur Verfügung stehenden Ressourcen immer erforderlich. Dabei ist nach Kitcher nicht davon auszugehen, dass jede dieser Entscheidungen gleich gut wäre, da sie jeweils zu einem Wissenszuwachs führten, nur in anderen Bereichen. Der Wert der Wissensgewinnung ist für ihn nicht allen anderen Werten übergeordnet und kein reiner Selbstzweck, sondern sollte durch demokratische Prinzipien reguliert werden.

WOS ist eine Wissenschaft, deren Ausrichtung sich in Bezug auf ihre politische Bedeutung verantwortungsvoll verhält. Idealerweise würden in dieser Wissenschaft Entscheidungen über die Ausrichtung von Forschung (via die Verteilung von Ressourcen), die ethische Beschränkung von Methoden sowie die Anwendung von Ergebnissen durch eine Konsensfindung der Repräsentanten verschiedener gesellschaftlicher Interessen gefällt. Diese Repräsentanten sind als ideale Deliberatoren konzipiert, die angeleitet durch unparteiische Experten - sowohl hinreichend Informationen über den Stand der Forschung und die bisherige Entwicklung bereichsspezifischer Signifikanzgraphen verarbeiten können als auch die Interessen der anderen Parteien in ihre Entscheidung einbeziehen (vgl. ebd., Kap. 10).

Ein solches Verfahren ist natürlich nicht praktisch durchführbar. WOS ist als normatives Ideal gedacht, dem Wissenschaft sich anzunähern versuchen sollte. Allerdings, so Kitcher, sollte diese Annäherung nicht auf die prozedurale Verwirklichung einer idealen deliberativen Konsensfindung zielen, sondern darauf, Ergebnisse hervorzubringen, die den Ergebnissen des idealen Prozederes glichen:

For perfectly well-ordered science we require that there be institutions governing the practice of inquiry within the society that invariably lead to investigations that coincide [...] with the judgments of ideal deliberators, representative of the distribution of viewpoints in society. [...] there's no thought that well-ordered science must actually institute the complicated discussions I've envisaged. The thought is that, however inquiry proceeds, we want it to match 
the outcomes those complex procedures would achieve at the points I've indicated. (Ebd., S. 122 f.)

Der Einwand liegt nahe, nach der Möglichkeit eines Vergleichs der Wirklichkeit mit den Ergebnissen idealer, nicht reproduzierbarer Verfahren zu fragen. Wie bestimmen wir die Ergebnisse dieser Verfahren, denen wir uns annähern sollen, ohne eine Möglichkeit sie durchzuführen? Diese strukturelle Konzeption einer Evaluierung, die auf einem Ideal auf der Prozessebene beruht, dieses Ideal jedoch mit Ergebnissen auf der Produktebene abzugleichen zielt, ist zweifelhaft. Kitcher zufolge erfordert WOS auf der Prozessebene, dass Institutionen entwickelt werden, die in etwa die Ergebnisse einer idealen Deliberation hervorbrächten (vgl. ebd., S. 123); auch deren Evaluierung ist demnach von dieser strukturellen Schwierigkeit betroffen. Zwar benennt er einige Punkte, in denen die gegebene Wissenschaft von einer wohl-geordneten abweiche, etwa die Vernachlässigung bestimmter Interessensgruppen oder die zunehmende Ausrichtung von Forschung nach kommerziellen Motiven. In Fällen, die der Idee einer demokratisch akzeptablen Agenda-Setzung offensichtlich widersprechen, mag eine solche Identifizierung möglich sein. Es ist jedoch problematisch vorauszusetzen, die Ergebnisse der idealen Deliberation würden, auch in komplexeren Situationen, stets mit unseren diesbezüglichen Intuitionen übereinstimmen. Die normative Fruchtbarkeit von Kitchers Ideal ist zumindest fragwürdig, da die Operationalisierbarkeit und Realisierbarkeit einer Annäherung an WOS unklar sind. ${ }^{43}$

Eine Diskussion über normative Fruchtbarkeit würde jedoch zunächst voraussetzen, dass die argumentativen Grundlagen des Ideals kohärent und überzeugend sind, was im Folgenden noch genauer untersucht werden soll. Insgesamt ist die Position Kitchers am eher konservativen Ende im Spektrum der Sozialepistemologie einzuordnen. In der Praxis ist Wissenschaft ein sozialer Prozess. Um diesen effektiv zu gestalten, etwa in Bezug auf die Arbeitsorganisation und Ressourcendistribution, sind soziale

\footnotetext{
${ }^{43}$ Ratsam scheint hier vielmehr eine Annäherung an den idealen Deliberationsprozess auf der Prozessebene, die auch auf dieser Ebene evaluiert wird. Diese könnte beispielsweise auf einen Einbezug von Laienperspektiven und verschiedenen Interessensgruppen und die Umsetzung des Ideals durch partizipative Verfahren zielen. Damit wären zumindest Schritte für eine Annäherung identifizierbar und auch in einem gewissen Grad realisierbar.
} 
Mechanismen und Normen nötig (vgl. dazu auch ders. 1993). Diese sozialen Bedingungen sind aber nicht entscheidend für die Beurteilung wissenschaftlicher Geltungsansprüche. Von der herkömmlichen Wissenschaftstheorie unterscheidet Kitcher sich vor allem dadurch, dass er traditionell eher der Wissenschaftssoziologie zugeordnete Themenbereiche zu einem Gegenstand der philosophischen Reflexion macht. Sein Ideal einer demokratischeren Wissenschaft bezieht sich auf die Ausrichtung und Anwendung von Forschung, nicht auf deren Rechtfertigung. Die neopositivistische Kontextunterscheidung wird beibehalten, die disziplinäre Relevanz der verschiedenen Kontexte allerdings neubewertet. Deshalb stellt Kitchers Ideal keine genuine Alternative zu dem Ideal der Wertfreiheit dar, sondern eher eine Ergänzung zu dessen zeitgenössischer Formulierung.

Seine Position basiert dabei auf einer Zurückweisung der Unterdeterminierungsthese, die problematisch scheint, insbesondere im Hinblick auf die angenommene Irrelevanz transienter Unterdeterminierung. Im weiteren Verlauf werde ich dafür argumentieren, dass Kitchers eigene Argumente in Bezug auf die Perspektiv- bzw. Zielgebundenheit von Klassifikationssystemen und Bewertungsstandards sowie für die historische Dimension des Signifikanzbegriffs weitreichendere Konsequenzen für die Wertfreiheit der Wissenschaft haben, als er selbst annimmt.

\subsection{Helen Longino: Social Value Management}

Helen Longinos kritischer kontextueller Empirismus steht in der Tradition der feministischen Erkenntnistheorie empiristischer Ausrichtung und unterscheidet sich von Kitchers Ansatz durch eine weitergehende Abweichung von traditionellen wissenschaftstheoretischen Positionen. Sie geht davon aus, dass Wissenschaft nicht nur kontingenterweise, sondern essentiell ein sozialer, d. h. durch Interaktion gekennzeichneter Prozess ist. Deshalb sei der soziale Rahmen, in welchem Wissenschaft stattfinde, nicht nur relevant in Bezug auf eine mögliche Optimierung etwa der Verteilung finanzieller oder humaner Ressourcen, sondern habe auch eine normative Bedeutung für wissenschaftstheoretische Kernbegriffe wie Objektivität 
oder Wissen. Die soziale Dimension von Forschung könne daher auch im Bezug auf wissenschaftliche Inhalte und ihre Bewertung nicht ausgeklammert werden, was jedoch nur dann Probleme generiere, wenn sozial und rational als dichotome Gegensätze verstanden würden. Damit zusammenhängend weist Longino die Idee einer wertfreien Wissenschaft zurück, wobei sie (anders als Kitcher) nicht nur Werteinflüsse auf die Ausrichtung von Forschung, sondern auch auf Inhalte und Evaluationskriterien als unter Umständen legitim erachtet.

Ausgangspunkt des kontextuellen Empirismus ist die Annahme einer Unterdeterminierung von Theorien, welche über die bloße Generalisierung von Beobachtungen hinausgehen und auf das Verständnis zugrunde liegender Prozesse und Entitäten zielen. Einerseits sei nicht eindeutig, anhand welcher Konsequenzen eine Hypothese zu überprüfen ist; andererseits bestehe die Möglichkeit, dass dieselben Daten verschiedene, auch inkompatible Hypothesen (oder Theorien) stützen. Deshalb sei die Beziehung zwischen theoretischen Hypothesen und empirischen Konsequenzen nicht als eine direkte, sondern als eine unterdeterminierte und durch Hintergrundannahmen vermittelte zu verstehen. Diese Hintergrundannahmen könnten sowohl methodologischer als auch substantieller Art sein und enthielten beispielsweise Annahmen über Kausalitätsbeziehungen oder ontologische Strukturen eines Gegenstandsbereichs. Entscheidend ist, dass diese zwischen Theorie und Empirie vermittelnden Hintergrundannahmen ihr zufolge wertbeladen sein können (vgl. Longino 1990, Kap. 3).

Exemplarisch sei an dieser Stelle ein bekanntes Beispiel aus der evolutionären Anthropologie erwähnt, in welchem es um die Erklärung der Entwicklung des Werkzeuggebrauchs geht. Hier bilden Fossilien von Knochen, Fußabdrücken oder Werkzeugen (neben nicht gerade unproblematischen Analogien zu zeitgenössischen Primaten und Jäger-und-SammlerVölkern) die (schmale) empirische Basis, welche jeweils zum Beleg für inkompatible Hypothesen angeführt wird. Im man-the-hunter Modell wird der Gebrauch von Werkzeugen mit den Erfordernissen der Jagd in Verbindung gebracht. Dadurch wird ein traditionell hauptsächlich männlicher Tätigkeitsbereich zu der Aktivität, die entscheidend den Fortschritt der Menschheit beeinflusste. In den 1970ern wurde von einigen Wissenschaftlerinnen das dem entgegengesetzte women-the-gatherer-Modell entwickelt, welches den Werkzeuggebrauch auf das Sammeln, Ausgraben und 
Zubereiten vorwiegend pflanzlicher Nahrung zurückführt. Allein die empirischen Funde reichen in diesem Fall nicht aus, ein Modell vor dem anderen auszuzeichnen; vielmehr setzt ihre Anführung als Evidenz jeweils bereits bestimmte Hintergrundannahmen voraus, wie etwa, dass Männer auf die Jagd gingen und Frauen derweil für den Haushalt sorgten. Auffällig ist, dass beide Modelle mit herkömmlichen geschlechtsspezifischen Rollenbildern konform gehen. Zudem scheint plausibel, dass die Zuordnung des Werkzeugsgebrauchs zu dem als männlich betrachteten Gebiet durch wertbeladene Annahmen unterstützt wird, die im Gegenmodell nicht geteilt werden (vgl. Brown 2001, S. 201 ff; Longino 1990, S. 106 ff; Longino/ Doell 1983).

Longinos Argumentation setzt dabei keine starke Interpretation der Unterdeterminierungsthese im Sinne vollständiger empirischer Äquivalenz voraus, sondern betrifft (wie bei Kuhn) auch Fälle, in denen die für rivalisierende Theorien angeführte Evidenz sich nicht vollständig deckt, sondern diese Theorien in Bezug auf unterschiedliche Teilaspekte ihre jeweiligen Stärken und Schwächen haben. Es geht ihr an dieser Stelle zudem nur darum, die Möglichkeit von Werteinflüssen via Hintergrundannahmen zu etablieren. Diese Möglichkeit ergibt sich prinzipiell bereits an dem Punkt, an dem von dem deterministischen Bestätigungsmodell des Neopositivismus abgerückt wird (was, wie dargestellt, zeitgenössisch dem philosophischen Mainstream entspricht).

One of the main lessons of the underdetermination argument is that there are no formal rules, guidelines, or processes that can guarantee that social values will not permeate evidential relations. If this is so, then it is a contingent matter whether a given theory produced according to the best rules is value-laden or not. There are two consequences: it can be asked about any given theory whether it is value-laden and, if so, with which values; and it becomes imperative to produce an analysis of objectivity (understood as social value management rather than absence of social values) that does not invoke formal rules. (Longino 2002a, S. 50)

Longino behauptet also nicht, dass alle Wissenschaft notwendig wertbeladen sei, sondern dass es aufgrund der prinzipiellen Möglichkeit wertbeladener Hintergrundannahmen unmöglich sei, Wertfreiheit durch wissen- 
schaftliche Verfahren generell zu garantieren. ${ }^{44}$ Ebenso ist ihre Argumentation damit vereinbar, dass bestimmte Bereiche (die eine größere Relevanz für soziale Fragen aufweisen) anfälliger für Werteinflüsse sind als andere. Andererseits ist es nach Longino nicht so, dass die Wertbeladenheit gleichbedeutend mit schlechter Wissenschaft ist und zur Aufgabe des Objektivitätsanspruchs führen muss. Vielmehr sei ein neues Verständnis von Objektivität erforderlich, das die Rolle von Hintergrundannahmen berücksichtige. Diese Rolle erinnert an Kuhnsche Paradigmen, da Hintergrundannahmen vorstrukturieren, welche Fragen gestellt werden, was als mögliche Lösung gilt und was als empirische Prüfung welcher Hypothesen betrachtet wird. Hintergrundannahmen seien jedoch prinzipiell isolierbar und artikulierbar und sollten durch einen Prozess der gegenseitigen Kritik innerhalb der wissenschaftlichen Gemeinschaft sichtbar gemacht werden. ${ }^{45}$

44 Kritiker richten sich oftmals gegen Longinos vermeintliche Annahme einer notwendigen Wertbeladenheit aller Wissenschaft (vgl. z. B. Ruphy 2006, S.191 f.: "the charge is essentially about the theoretical impossibility of value-neutral results"; Haack 1996, S. 88: "Longino [is] committed to the thesis that social values are inextricable from science". Diese Interpretation halte ich für verfehlt. Es geht Longino nicht um die Unmöglichkeit von Wertfreiheit, sondern vielmehr darum, dass Wertfreiheit auch durch die Einhaltung wissenschaftlicher Standards nicht immer garantiert werden kann (vgl. auch Rolin 2002). Derartige irreführende Interpretationen stehen häufig im Zusammenhang mit einer generellen Ablehnung der Idee einer feministischen Erkenntnistheorie. Diese Ablehnung begründet sich nicht nur in einer Sorge um den Verlust der epistemischen Funktion des Wertfreiheitsideals, sondern auch um seine politische Funktion, beispielsweise sexistischen Vorurteilen durch Forschung den Boden zu entziehen. Entsprechend wird argumentiert, dass feministische Erkenntnistheorie nicht nur Objektivität, sondern damit zugleich den Feminismus untergräbt (vgl. Haack 1996, Koertge 2000). Wie noch dargestellt werden soll, läuft Longinos Ablehnung des Wertfreiheitsideals jedoch nicht auf die Unmöglichkeit einer Kritik sexistischer Werteinflüsse in Theorien hinaus. Allerdings sei angemerkt, dass es innerhalb der feministischen Erkenntnistheorie große Unterschiede gibt. Ich stimme Haack zu, wenn sie Ansätze, die z. B. eine besondere kognitive Natur der Frau postulieren und weibliches Wissen der männlichen Wissenschaft gegenüberstellen, als ausgesprochen sexistisch kritisiert.

45 Diese permanente Artikulation und Diskussion grundlegender strukturierender Prämissen ist natürlich enorm aufwendig. Gerade die Konsensfähigkeit paradigmatischer Annahmen stellte bei Kuhn die Voraussetzung für den Fortschritt in nicht-revo- 
Ebendieser Prozess der Kritik bildet die Basis der alternativen Konzeption von Objektivität, die Longino vorschlägt. Durch die gegenseitige Kritik könnten implizite Werthaltungen aufgedeckt und individuelle Präferenzen der Wissenschaftler gradweise ausgesiebt werden. Allerdings gebe es kein natürliches Ende dieses Prozesses, da auch diese Kritik wiederum von Hintergrundannahmen abhängig sei. Objektivität nach Longino ist daher nicht gleichzusetzen mit der Eliminierung von Werteinflüssen durch den sozialen Prozess der Kritik, da das Erreichen einer solchen Eliminierung nicht identifizierbar wäre - die Möglichkeit weiterer Werteinflüsse ließe sich nie ganz ausschließen. Im Gegenteil entkoppelt sie den Objektivitätsbegriff von dem Ideal der Wertfreiheit. Die für Longino relevante Ebene von Objektivität ist diejenige, die sich auf den Prozess der Produktion wissenschaftlichen Wissens bezieht. Dabei verlagert sie jedoch den Fokus von Methoden oder Regeln, die prinzipiell von Individuen anwendbar sind, auf deren Interaktion. Deshalb ist für sie die soziale Ebene des Forschungsprozesses konstitutiv für die Objektivität der Wissenschaft:

It is the possibility of intersubjective criticism [...] that permits objectivity in spite of the context dependence of evidential reasoning. [...] Objectivity, then, is a characteristic of a community's practice of science (Dies. 1990, S. 71, 74).

Objektiv ist die Praxis einer wissenschaftlichen Gemeinschaft, und sie ist es dann, wenn sie effektive Kritik ermöglicht. Hierfür stellt Longino vier Bedingungen auf, welche die Annäherung an das Ideal sozialer Objektivität gleichzeitig operationalisieren. Erstens müsse es etablierte Verfahren und Kanäle der Kritik geben (recognized avenues for criticism), z. B. funktionierende peer-review Prozesse bei wissenschaftlichen Zeitschriften, Diskussionsmöglichkeiten auf Konferenzen usw. Wichtig sei zudem auch die Anerkennung kritischer Arbeiten statt einer einseitigen Ausrichtung auf originelle Forschung. Zweitens müsse diese Kritik transformative Wirkung zeigen, d. h. die wissenschaftliche Gemeinschaft müsse sich mit ihr auch angemessen auseinandersetzen (community response oder uptake). Dies sei wiederum nur möglich auf der Basis geteilter Evaluierungskriterien (shared standards), welche die dritte Bedingung von Objektivität darstellen. Viertens fordert sie die gleichberechtigte Verteilung intellektueller

lutionären Phasen dar. Für Longinos Ansatz könnte dies, angesichts begrenzter Ressourcen, zumindest im Hinblick auf die praktische Umsetzbarkeit problematisch sein. 
Autorität unter allen entsprechend qualifizierten Teilnehmern und den Einbezug aller relevanten Alternativen in die Diskussion (tempered equality of intellectual authority). Sie geht davon aus, dass Werthaltungen oftmals mit einer großen Selbstverständlichkeit der damit verknüpften Ansichten und Urteile einhergehen, und geteilte Werte daher durch den Prozess der Kritik nicht oder nur schwer sichtbar gemacht werden können. Zudem setzt sie die (durchaus plausible empirische) Annahme voraus, dass diese Werte zu einem großen Teil mit dem sozialen und kulturellen Hintergrund der betreffenden Personen korrelieren. Je größer daher die Diversität dieser Personen (etwa in Bezug auf Schichtzugehörigkeit, kulturellen oder ethnischen Hintergrund, Religion, Geschlecht, politische Orientierung usw.), desto kleiner sei der gemeinsame und damit unsichtbare Nenner (vgl. ebd., Kap. 4).

Wie Kitchers WOS integriert damit auch Longinos SVM egalitäre demokratische Elemente: wenn Werte Einfluss auf die Wissenschaft nehmen, sollten diese Werte nicht einseitig politische Machtstrukturen widerspiegeln. Allerdings ist das für sie nicht nur ein politisches, sondern auch ein epistemisches Erfordernis hinsichtlich der Sichtbarkeit vorhandener Werteinflüsse. Sozial und rational seien entsprechend nicht als Gegensätze zu denken; stattdessen werde die soziale Ebene des Forschungsprozesse optimalerweise zu einer epistemischen Ressource. Insbesondere die Forderung nach Diversität und intellektueller Gleichberechtigung erinnert dabei an Jürgen Habermas Theorie und Ideal kommunikativen Handelns. Im Gegensatz zu Habermas, für den dieses Ideal auf der prozeduralen Ebene mit Wahrheit auf der Produktebene verknüpft ist, ist soziale Objektivität zwar eine Bedingung, jedoch nicht entscheidend für die Geltungsansprüche der Resultate (vgl. ebd., S. 197 ff.).

Longino versteht Objektivität demnach als wesentlich soziales und prozedurales Kriterium. Dieser Objektivitätsbegriff erfordert weder eine Unparteilichkeit der beteiligten Individuen, ${ }^{46}$ noch ist er mit dem Ergebnis des Prozesses der gegenseitigen Kritik gleichzusetzen. ${ }^{47}$ Sie löst damit die

\footnotetext{
${ }^{46}$ Sie fordert von den Individuen die Teilnahme an dem Prozess der Kritik; dies muss jedoch keine letztliche Abhängigkeit von einer Objektivität Einzelner bedeuten, sofern dies durch soziale Mechanismen (wie etwa Reputationszuweisung) geregelt würde.

${ }^{47}$ Im Gegensatz dazu vertritt etwa Miriam Solomon eine konsequentialistische Auffassung, die sich auf das Ergebnis der gegenseitigen Aufhebung individueller Wertein-
} 
von Fine (1998) kritisierten Gleichsetzungen der verschiedenen Ebenen des Objektivitätsbegriffs auf und ermöglicht dadurch die Loslösung wissenschaftlicher Objektivität von der Voraussetzung der Wertfreiheit.

Eine häufige Kritik an diesem Objektivitätsverständnis lautet, dass es relativistisch sei, worauf hier kurz exemplarisch eingegangen werden soll: ${ }^{48}$ "Essentially, what social objectivity delivers is merely a bridled relativism. At core, it is no more objective than the unbridled relativism that Longino wishes to avoid" (Smith 2004, S. 145). Dieser Vorwurf resultiert aus einem Missverständnis von Longinos Kontextualismus als Konsenstheorie in Bezug auf die Frage der Rechtfertigung:

Knowledge certainly does often benefit from the contributions of many people. It does not follow from this, however, that others' beliefs should become the standard of objective knowledge. Yet this is the implication of the social conception, which replaces the relevant reality as the arbiter of knowledge with a group's critical consensus. (Ebd., S.148)

Der kontextuelle Empirismus zielt jedoch nicht darauf, Evidenz durch Konsens zu ersetzen, sondern geht von der Unmöglichkeit aus, der Realität die Rolle einer unparteiischen Prüfinstanz zuzuschreiben. Diese Unmöglichkeit erklärt sich aus der Perspektivgebundenheit unserer Repräsentationen dieser Realität, die auf der von Longino postulierten Rolle von Hintergrundannahmen beruht. Dennoch ist weder jedes Resultat ein möglicher Konsens, noch ist Konsens allein hinreichend für Objektivität (des Prozesses) oder Gültigkeit (der Ergebnisse). Entscheidend ist hier, dass die Bedingungen effektiver Kritik geteilte Standards enthalten, die unter anderem empirische Adäquatheit zu einer notwendigen Bedingung der Theoriewahl machen.

Während Longino Objektivität als prozedurale Eigenschaft wissenschaftlicher Gemeinschaften versteht, benutzt sie den Begriff des Wissens, um zwischen erfolgreichen und erfolglosen wissenschaftlichen Inhalten zu unterscheiden. Ihr Wissensbegriff ${ }^{49}$ erfordert dabei einerseits eine hinrei-

flüsse auf der Ebene der Gemeinschaft bezieht (vgl. Solomon 2001; siehe dazu auch Wilholt 2009b).

${ }^{48}$ Siehe auch Büter (2010).

${ }^{49}$ Auch wissenschaftliches Wissen ist für Longino essentiell sozial, da es auf wesentlich durch Interaktion bestimmten kognitiven Prozessen beruhe. So sei etwa Beobachtung nicht mit individuellen Wahrnehmungen $\mathrm{zu}$ identifizieren, sondern mit 
chende Übereinstimmung von Theorie und Empirie, andererseits das Überstehen des kritischen Prozesses in einer als sozial objektiv charakterisierbaren Gemeinschaft. Dadurch leistet er eine Integration von Normen der Rechtfertigung und Normen der Interaktion, d. h. der rationalen und der sozialen Ebene von Forschung.

Some content $\mathrm{A}$ is epistemically acceptable in community $\mathrm{C}$ at time $\mathrm{t}$ if $\mathrm{A}$ is or is supported by data $\mathrm{d}$ evident to $\mathrm{C}$ at $\mathrm{t}$ in light of reasoning and background assumptions which have survived critical scrutiny from as many perspectives as are available to $\mathrm{C}$ at $\mathrm{t}$, and $\mathrm{C}$ is characterized by venues for criticism, uptake of criticism, public standards, and tempered equality of intellectual authority. [...] A given content, $\mathrm{A}$, accepted by members of $\mathrm{C}$ counts as knowledge for $\mathrm{C}$ if $\mathrm{A}$ conforms to its intended object(s) (sufficiently to enable members of $\mathrm{C}$ to carry out their projects with respect to that/those object(s)) and A is epistemically acceptable in C. (Longino 2002a, S. 135 f.)

Durch den Begriff conformation ersetzt Longino die Evaluation wissenschaftlicher Inhalte als wahr oder falsch durch die Evaluation des Erfolgs von Beiträgen $\mathrm{zu}$ bestimmten Forschungszielen. Wie Kitcher illustriert auch Longino ihre Auffassung des Erfolgs wissenschaftlicher Inhalte durch eine Analogie zu Karten: ihre Qualität bemesse sich danach, inwieweit sie den betreffenden Gegenstand in der Hinsicht erfolgreich abbildeten, die sie ihren spezifischen Zweck erfüllen lasse. Ein Vorteil der conformationKategorie liege dabei darin, dass diese sich unproblematisch auf verschiedene Formen der Repräsentation von Inhalten anwenden ließe, also nicht nur auf Theorien als Mengen von Sätzen oder Propositionen, sondern auch auf Modelle, visuelle Repräsentationen usw. (vgl. ebd., S. 108-121; siehe auch Kitcher 2002a).

Epistemisch akzeptierbares Wissen wäre dabei nicht notwendigerweise wertfrei: auch wertbeladenes Wissen könnte in einem objektiven Prozess effektiver Kritik entstanden sein. Durch die Forderung nach einer (zielspezifischen) hinreichenden Übereinstimmung mit der Empirie und geteilten Standards der Kritik soll andererseits jedoch eine direkte Folgerung vom Sollen auf das Sein ausgeschlossen werden. Des Weiteren ist diese Auffassung mit einem Pluralismus in Bezug auf erfolgreiche wissen-

durch interaktive Prozesse stabilisierten, geteilten und wiederholbaren Wahrnehmungen, ebenso wie wissenschaftliches Argumentieren und Schlussfolgern prinzipiell dialogischer Natur sei (vgl. dies. 2002a, Kap. 5). 
schaftliche Inhalte vereinbar. Wie gesagt ist dieser Pluralismus weitreichender als bei Kitcher, da Longino auch die Möglichkeit inkompatibler, jeweils erfolgreicher Inhalte zulässt. Dies beruht vor allem darauf, dass sie auch in Bezug auf die Standards der Kritik eine pluralistische Position vertritt. Wie Kitcher geht sie davon aus, dass ein Ziel wie Wahrheit oder Wissen zu unspezifisch ist, um konkrete Forschung anzuleiten. Stattdessen nimmt sie bereichsspezifische Ziele einzelner Forschungsprogramme an, die wiederum auch mit Werthaltungen verbunden sein könnten, z. B. genderspezifisches Verhalten biologisch zu erklären oder genderspezifische Diskriminierung in der Wissenschaft sichtbar zu machen. Statt zwischen kognitiven und nicht-kognitiven Werten unterscheidet sie zwischen kontextuellen und konstitutiven Werten, wobei konstitutiv die Werte sind, welche die jeweiligen Ziele befördern sollen. Aus derartigen Zielen könnten nun bestimmte, z. B. feministisch motivierte Werte für die Theoriewahl abgeleitet werden, die jedoch gleichzeitig eine legitime kognitive Rolle hätten (vgl. Longino 1995, 1996).

Insgesamt räumt der kritische kontextuelle Empirismus Werteinflüssen eine wesentlich größere Rolle ein als Kitchers moderater Realismus. Das Ideal der Wertfreiheit ist für Longino nicht nur in Bezug auf diejenigen Aspekte problematisch, die traditionell als von der Rechtfertigung von Theorien unabhängig gelten. Vielmehr bezweifelt sie gerade auch das Element, welches oben als notwendige Bedingung der Wertfreiheit beschrieben wurde: die Trennbarkeit kognitiver und nicht-kognitiver Werte bei der Theoriewahl. Insbesondere diese These einer kontextabhängigen Legitimität von Theoriewahlkriterien ist entsprechend kontrovers und wird unten noch ausführlich diskutiert (siehe Kapitel 7). Dabei wird auch der Frage nachzugehen sein, ob Longinos Pluralismus in Bezug auf evaluative Standards ein Problem für ihren sozialen Objektivitätsbegriff darstellt. Die mit diesem verknüpfte Forderung nach einer möglichst diversen Kritik scheint zumindest in einer gewissen Spannung zu der Annahme zu stehen, dass die Grundlagen dieser Kritik abhängig von einzelnen wissenschaftlichen Gemeinschaften und deren spezifischen (möglicherweise auch wertbeladenen) Zielen sind. Wie kann auf dieser Basis eine effektive gemeinschaftsübergreifende Kritik ermöglicht werden, d. h. eine Kritik aus verschiedenen Perspektiven, die bestimmte Werthaltungen gerade nicht teilen? 
Neben der Bedingung einer Unterscheidbarkeit kognitiver und nichtkognitiver Werte zieht die von Longino konstatierte Rolle von Hintergrundannahmen und deren Einfluss auf die Charakterisierung von Gegenstandsbereichen dabei eine weitere Voraussetzung des zeitgenössischen Wertfreiheitsideals in Frage, dass nämlich Werteinflüsse des Entdeckungszusammenhangs in ihrer Wirkung auf diesen beschränkt werden können. Hintergrundannahmen beeinflussen bei Longino nicht nur die Auswahl von Hypothesen, sondern auch die Entscheidung darüber, woran diese überhaupt überprüft werden. Auch dieser Punkt soll im weiteren Verlauf noch geklärt und diskutiert werden.

Anders als Kitchers Ideal einer WOS, das als Ergänzung des Wertfreiheitsideals um eine normative Betrachtung des Entdeckungs- und Anwendungszusammenhangs beschrieben wurde, stellt Longinos SVM eine genuine Alternative dar. Es ist diesbezüglich wichtig zu sehen, dass ihre Neukonzeption von Objektivität nicht darauf zielt, sich der Wertfreiheit durch einen sozialen Prozess anzunähern, sondern vorhandene Werte sichtbar zu machen, um entsprechende Einflüsse demokratisieren zu können. Dieses demokratische Element begründet sich dabei nicht nur in der gesellschaftlichen Verantwortung von Wissenschaft, sondern auch in dem epistemischen Erfordernis, Selbstverständliches hinterfragbar zu machen. Wie gesagt argumentiert Longino andererseits nicht dafür, dass alle Wissenschaft notwendig wertbeladen sei; so ließe sich auch die Eliminierung idiosynkratischer Werte durchaus im Sinne einer Annäherung an Wertfreiheit verstehen. Der Punkt ist jedoch, dass sie eine Gleichsetzung wertfreier mit guter und wertbeladener mit schlechter Wissenschaft ablehnt. Die Frage ist ihr zufolge nicht, ob bestimmte Ergebnisse wertfrei seien, sondern ob etwaige Werteinflüsse in diesem spezifischen Fall legitim seien, d. h. ob diese Werteinflüsse mit den Bedingungen für soziale Objektivität vereinbar sind.

Zuletzt sei noch darauf hingewiesen, dass SVM und WOS nicht die einzigen sozialepistemologischen Alternativen zu dem Ideal der Wertfreiheit sind. So plädiert beispielsweise Janet Kourany für eine Socially Responsible Science. Sie geht dabei ebenfalls von der Möglichkeit von Werteinflüssen auch im Zusammenhang der Rechtfertigung aus und argumentiert, dass im Falle empirisch adäquater Alternativen die politisch progressivere gewählt werden sollte, wobei diese Progressivität im Sinne 
egalitärer demokratischer Werte zu verstehen sei. Anzumerken ist, dass dies nicht Longinos Position zur Theoriewahl entspricht: auch wenn diese Wahl ihr zufolge nicht zwingend wertfrei ist, erfordert der soziale Objektivitätsbegriff doch den Einbezug diverser Perspektiven und Werte. Problematisch an Kouranys Ansatz ist, dass dieser eine Entscheidung darüber voraussetzt, welches die richtigen Werte sind. Da diese Möglichkeit zumindest sehr strittig ist, werde ich ihren Ansatz im Folgenden nicht eingehender diskutieren. Kourany geht davon aus, dass ein hinreichender Konsens in Bezug auf egalitäre Werte besteht, um diese Entscheidungen zu treffen; auch das halte ich jedoch für sehr fraglich (gerade Beispiele der feministischen Erkenntnistheorie für androzentristische Forschung etwa zur weiblichen Intelligenz usw. scheinen eher das Gegenteil zu belegen). Selbst wenn eine Einigung in Bezug auf Wertfragen gegeben wäre, bliebe zudem offen, wie auf dieser Grundlage die epistemische Vertrauenswürdigkeit der Wissenschaft zu begründen wäre (vgl. dazu Kourany 2003a, 2003b sowie Giere 2003). ${ }^{50}$

Diese kursorischen Anmerkungen sind natürlich unzureichend für eine abschließende Bewertung des Ideals sozial verantwortlicher Wissenschaft. Dennoch werde ich mich vorwiegend auf die bisher einflussreichs-

\footnotetext{
${ }^{50}$ Außerdem nimmt Kitcher in seinem neuesten Buch (2011) einige Änderungen seiner hier dargestellten Position vor. Insgesamt räumt er nun Werten einen weitreichenderen Einfluss auf die Rechtfertigung ein, was er vor allem durch das Problem der induktiven Risiken begründet. Gleichzeitig vertritt auch er eine Position derart, dass diese Werteinflüsse von den richtigen Werten ausgehen sollten. Welches das sind, ließe sich naturalistisch bestimmen, indem der evolutionäre Beitrag von Werten zu sozialen Koordinierungsproblemen und einer entsprechenden Sozialtechnologie betrachtet werde. Dieser Möglichkeit (das Sollen aus dem Sein herzuleiten) stehe ich ebenfalls sehr skeptisch gegenüber. Zumindest scheint es aber aufgrund der Kontroversität einer solchen Position nicht ratsam, sie zur Grundlage eines wissenschaftsphilosophischen Ideals zu machen. Leider ist Kitchers Buch zu spät erschienen, um es in der vorliegenden Arbeit ausführlich zu diskutieren; ich werde mich daher auf die Darstellung wohlgeordneter Wissenschaft von 2001 konzentrieren.

Es sei noch erwähnt, dass auch Heather Douglas einen Alternativvorschlag zum Wertfreiheitsideal macht, der nicht auf einer Unterscheidung legitimer und illegitimer Werte beruht, sondern zwischen einer direkten und indirekten Rolle von Werten differenziert. Da ihr Ansatz wesentlich auf ihrer Argumentation zu induktiven Risiken beruht, werde ich ihn im entsprechenden Kap. 8 näher erläutern und diskutieren.
} 
ten Ansätze von Kitcher und Longino konzentrieren. Beide beziehen den sozialen Kontext von Wissenschaft in die wissenschaftsphilosophische Reflexion ein und verbinden diese mit einer demokratischen Zulassung verschiedener wertbeladener Perspektiven, unterscheiden sich jedoch eklatant hinsichtlich der legitimen Reichweite dieser Werteinflüsse. Entsprechend unterschiedlich stehen sie zur Frage der Wertfreiheit, weshalb eine Diskussion dieser beiden Alternativen geeignet scheint, Licht auf das Verhältnis von Wissenschaft und Werten zu werfen. Im folgenden Teil werden zunächst einige empirische Beispiele für diese Diskussion erarbeitet; in Teil III werden darauf aufbauend die einzelnen Argumente für und wieder das Wertfreiheitsideal eingehend diskutiert, um schließlich eine Bewertung der verschiedenen Idealformulierungen zu erlauben. 